\title{
Identification of lipid heterogeneity and diversity in the developing human brain
}

Aparna Bhaduri1,2,3,7* , Elizabeth K. Neumann ${ }^{4,5,7}$, Arnold R. Kriegstein ${ }^{1,2}$, Jonathan V. Sweedler ${ }^{4,5,6^{*}}$

${ }^{1}$ Department of Neurology, University of California, San Francisco (UCSF), San Francisco, CA, 94143, USA.

${ }^{2}$ The Eli and Edythe Broad Center of Regeneration Medicine and Stem Cell Research, UCSF, San Francisco, CA, 94143, USA.

${ }^{3}$ Department of Biological Chemistry, UCLA, Los Angeles, CA 90095, USA.

${ }^{4}$ Department of Chemistry, University of Illinois at Urbana-Champaign, Urbana, IL, 61801, USA.

${ }^{5}$ Beckman Institute for Advanced Science and Technology, University of Illinois at Urbana-Champaign, Urbana, IL, 61801, USA.

${ }^{6}$ Neuroscience Program, University of Illinois at Urbana-Champaign, Urbana, IL, 61801, USA.

${ }^{7}$ These authors contributed equally: Aparna Bhaduri, Elizabeth K. Neumann

${ }^{*}$ Corresponding Authors:

Aparna Bhaduri; ABhaduri@mednet.ucla.edu

Jonathan V. Sweedler; jsweedle@illinois.edu 
A

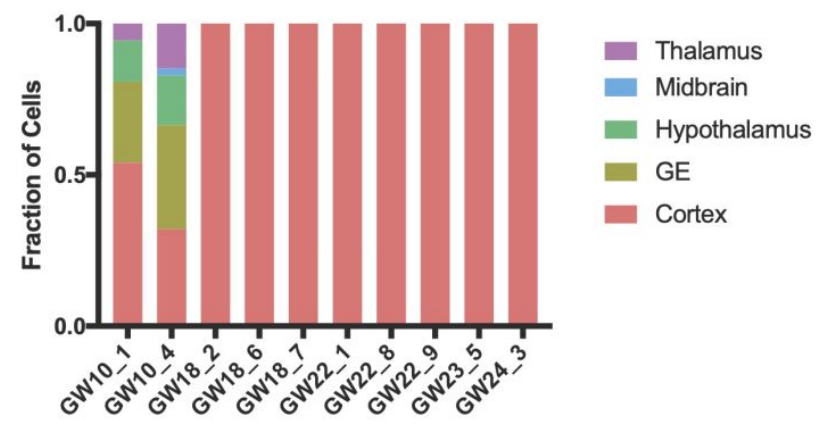

C Clusters By Sampled Ages
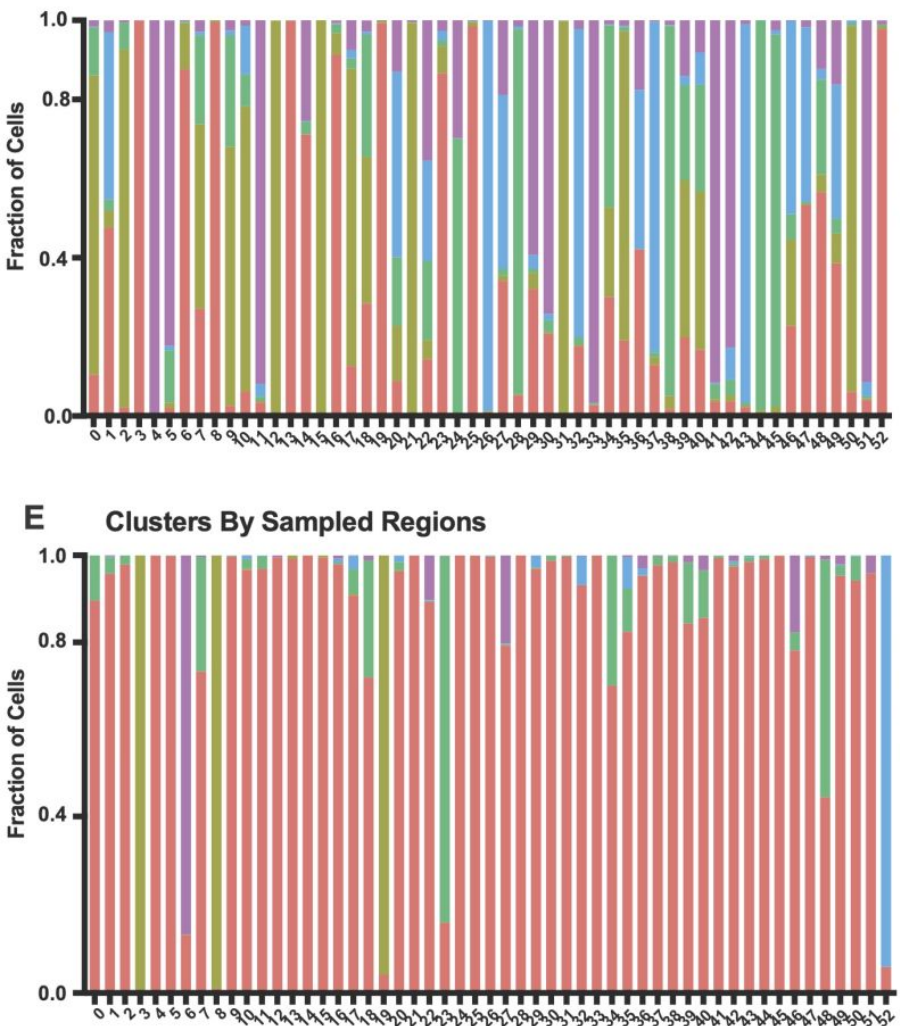

G

Lipid Composition Across Ages

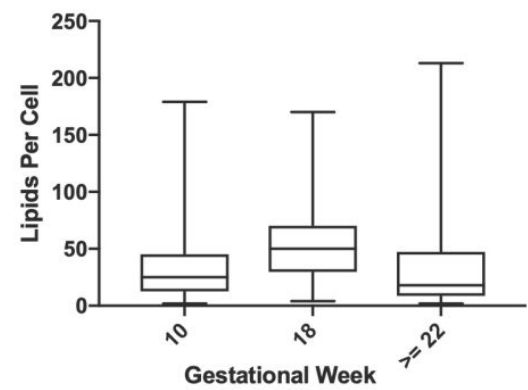

B

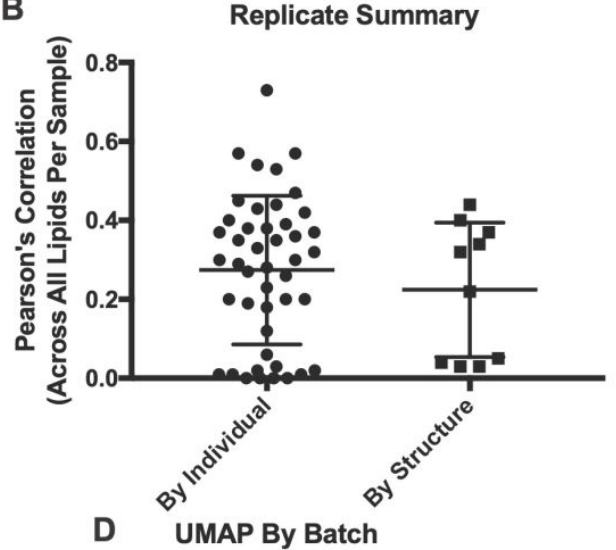

Age (GW)

24

23

22

18

10
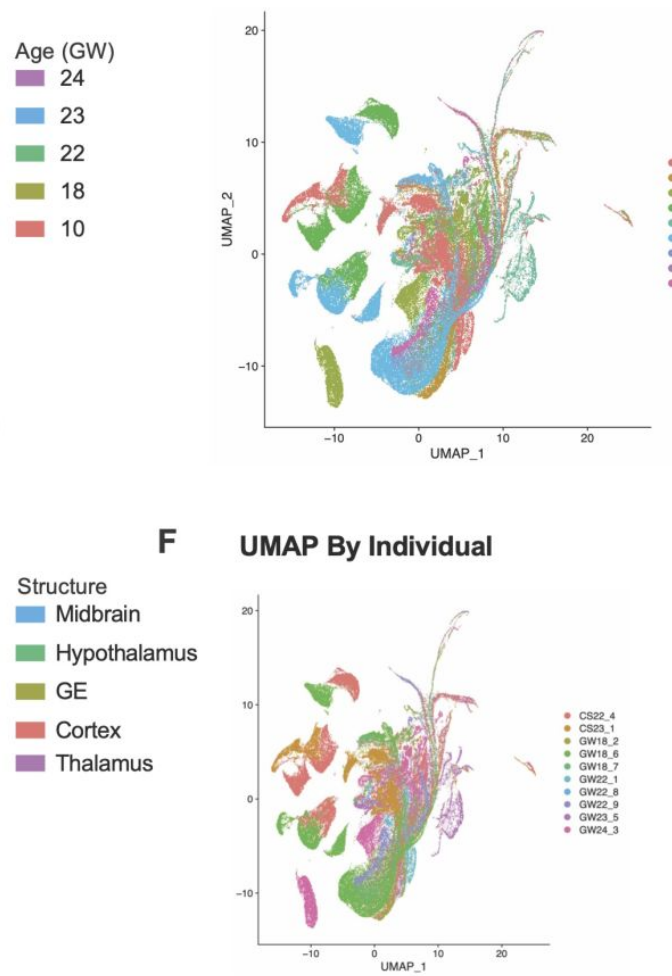

Figure S1. Heterogeneity of Lipids Across Developing Human Brain Stages and Regions. A) Stacked bar chart depicting the brain structures that were sampled from each individual sample. Individual samples labeling the $x$-axis include stage (Carnegie Stage (CS) or Gestational week (GW)) and a number to distinguish individuals from the same stages. B) Pearson's correlations were performed between the lipid values for each pair of individuals and structures. The range of correlations is shown in a box and whisker plot to indicate the level of replicability between experiments. Min to max is shown, with the bar at the mean and dots to indicate each individual comparison. C) Stacked bar chart shows the cluster level composition by age based upon the 
clustering shown in Figure 1b. D) UMAP plot colored by each batch sampled. Although some of the peripheral clusters are strongly enriched for one individual, the central UMAP and the bulk of the data shows strong intermixing among individuals within a cluster. E) Stacked bar chart shows the cluster level composition by brain structure based upon the clustering shown in Figure 1b. F) UMAP plot colored by each individual sampled. Although some of the peripheral clusters are strongly enriched for one individual, the central UMAP and the bulk of the data shows strong intermixing among individuals within a cluster. G) The number of lipids for each age sampled is shown in a box and whisker plot, from min to max with the bar at the mean. 

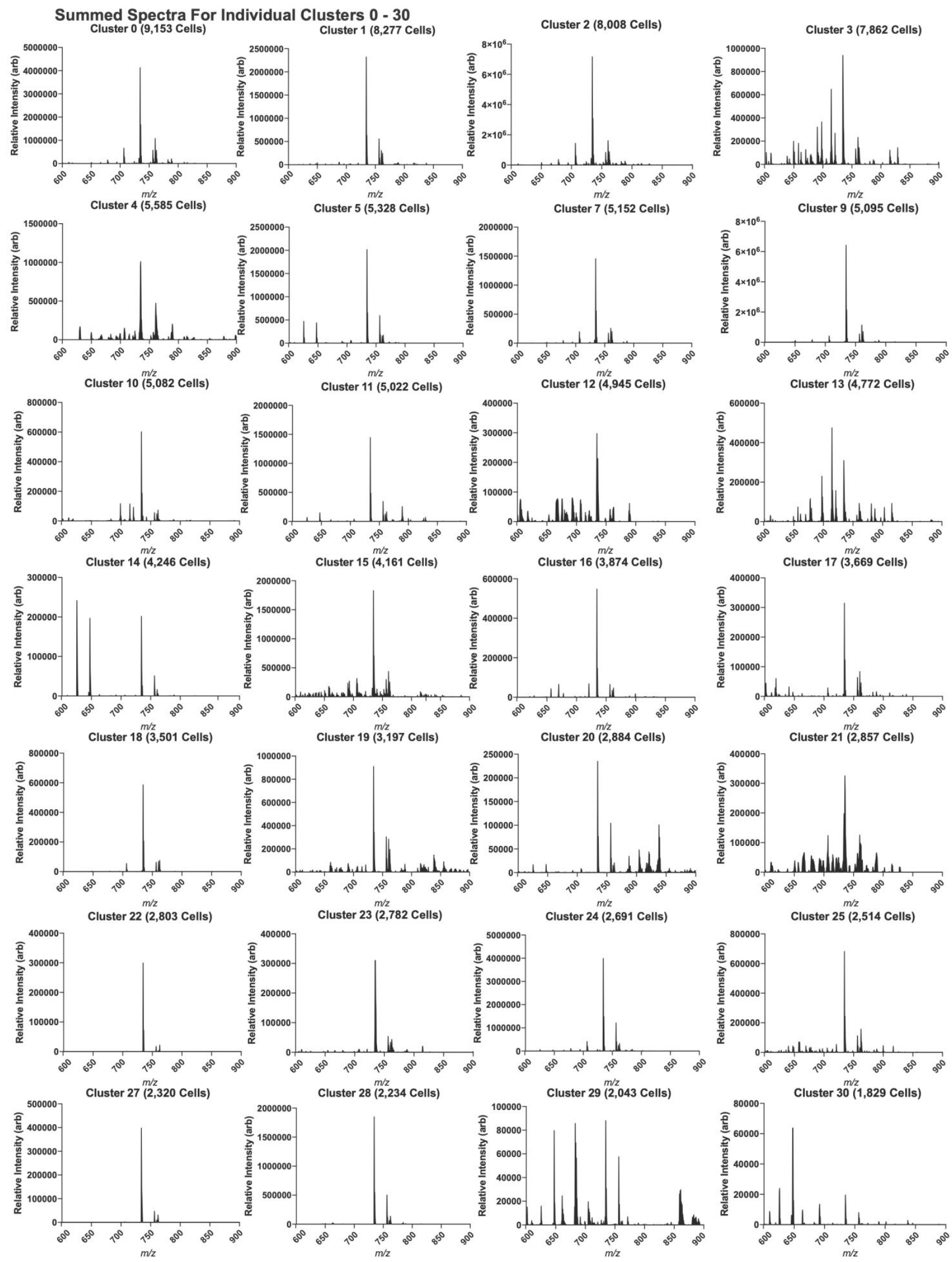

Figure

S2. Cluster Level Heterogeneity of Lipid Spectra Cluster 0 to 30 . Averaged lipid spectra from clusters 0 to 30 , excluding those shown in the main figures. In most cases, each spectrum is anchored by a base peak at 734.6, which has been identified as PC(32:0). 
Summed Spectra For Individual Clusters 31 - 52
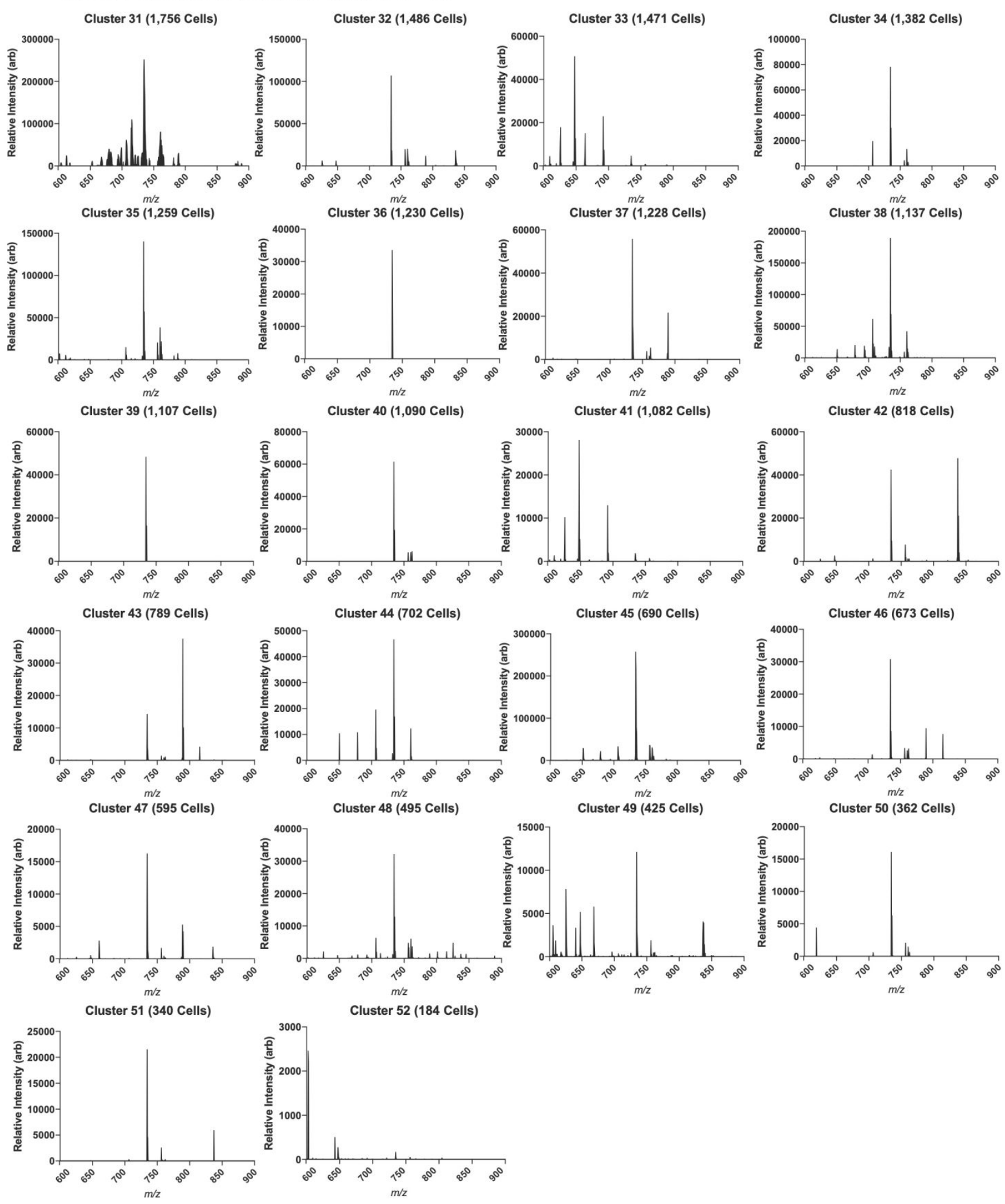

Figure

S3. Cluster Level Heterogeneity of Lipid Spectra Cluster $\mathbf{3 1}$ to $\mathbf{5 2}$. Averaged lipid spectra from clusters 31 to 52 , excluding those shown in the main figures. In most cases, each spectrum is anchored by a base peak at 734.6, which has been identified as PC(32:0). 
Feature Plots of Selected Cluster Enriched Lipids

$\mathrm{PC}(38: 3)$

Cluster 2

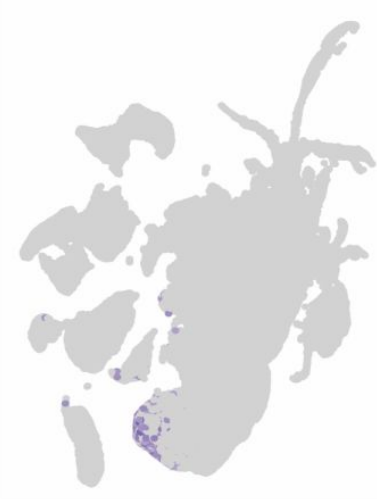

$$
\mathrm{PC}(36: 1)+\mathrm{H}
$$

Cluster 9

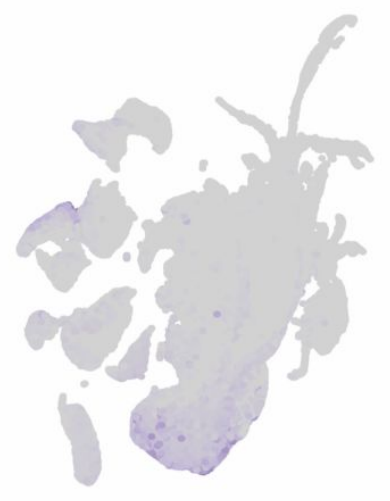

$\mathrm{PA}(\mathrm{O}-40: 0)+\mathrm{K}$ Cluster 24

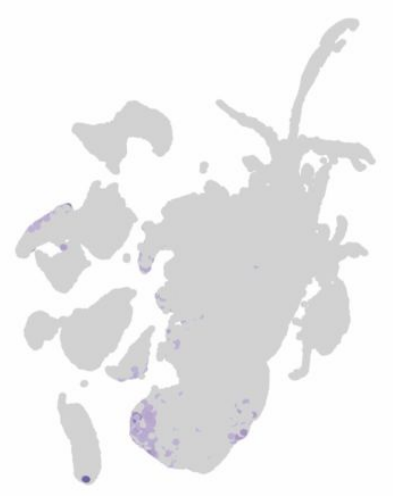

PA(32:3)

Cluster 3

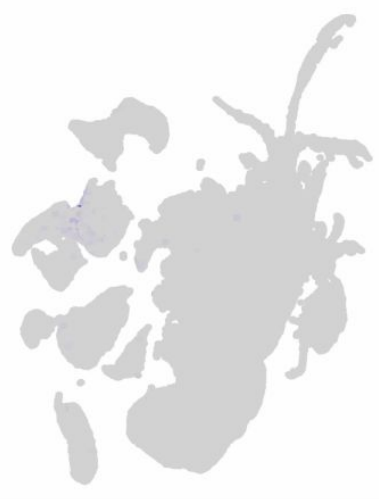

$\mathrm{PC}(36: 1)+\mathrm{K}$

Cluster 11

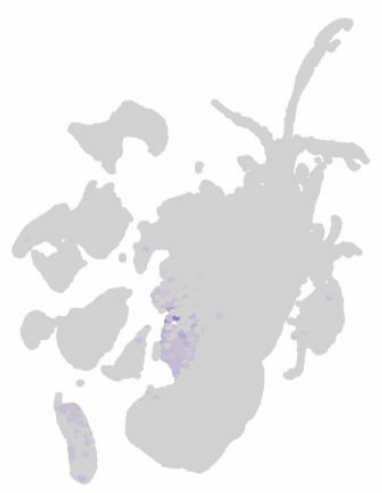

$\mathrm{SM}(\mathrm{d}-30: 0)$

Cluster 30

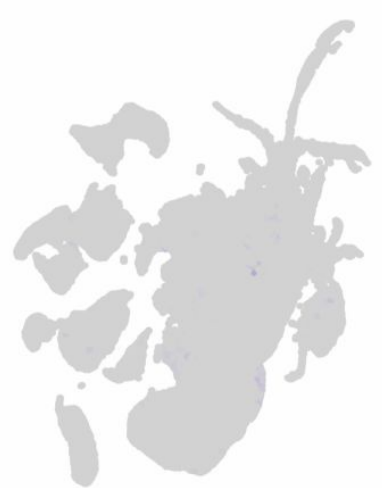

[TG(38:1)+H]+

Cluster 6

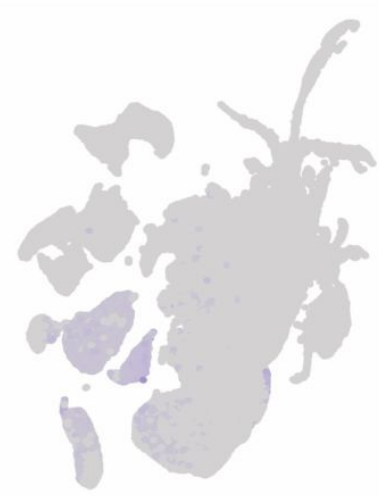

$\mathrm{PE}(\mathrm{O}-42: 6)$

Cluster 20

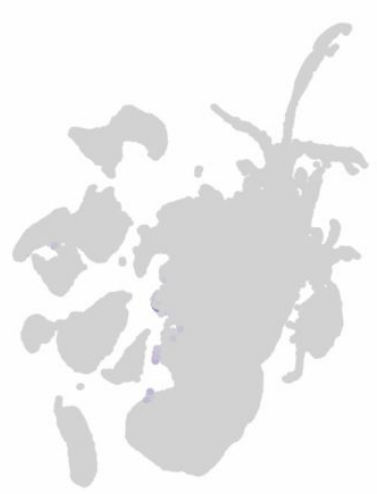

CerP(d34:0)

Cluster 31

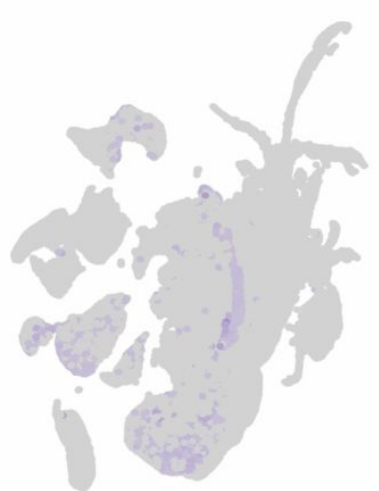

Figure S4. Feature Plots of Cluster Enriched Identified Lipids. Nine detected and identified lipids with cluster-level enrichments are shown here as feature plots in UMAP space, with more purple signal indicating more detection of that lipid in a cell. 

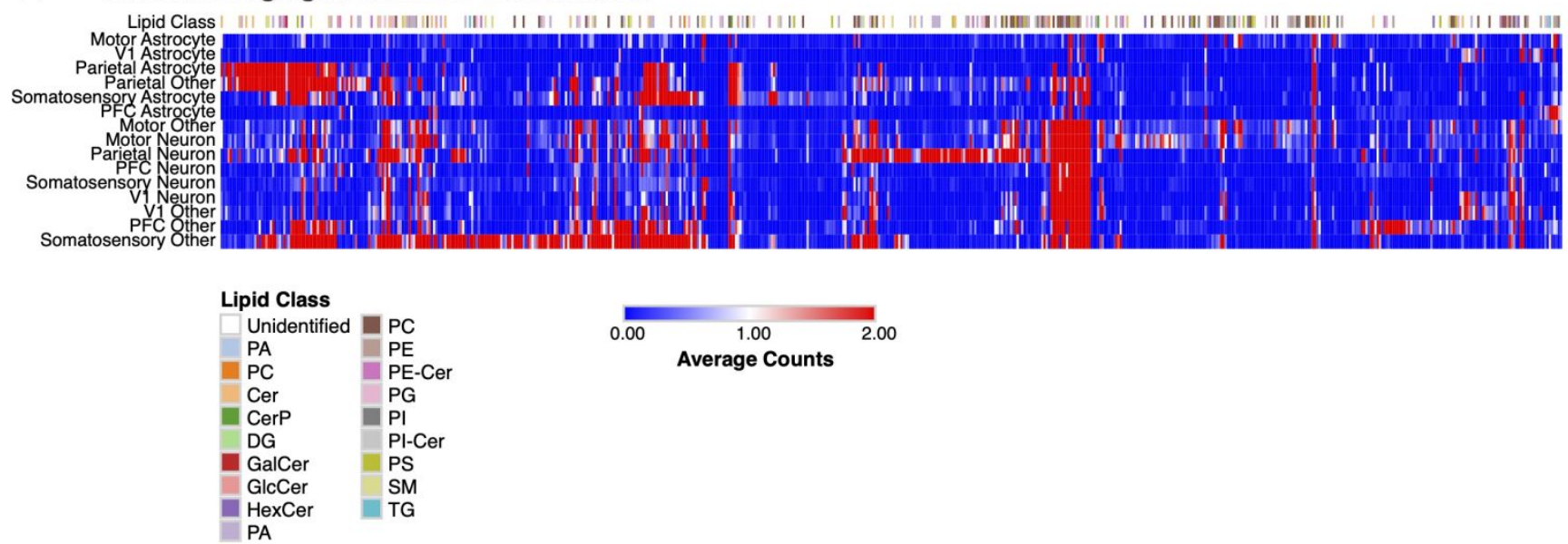

B Cell Type Segregation Across Brain Structures

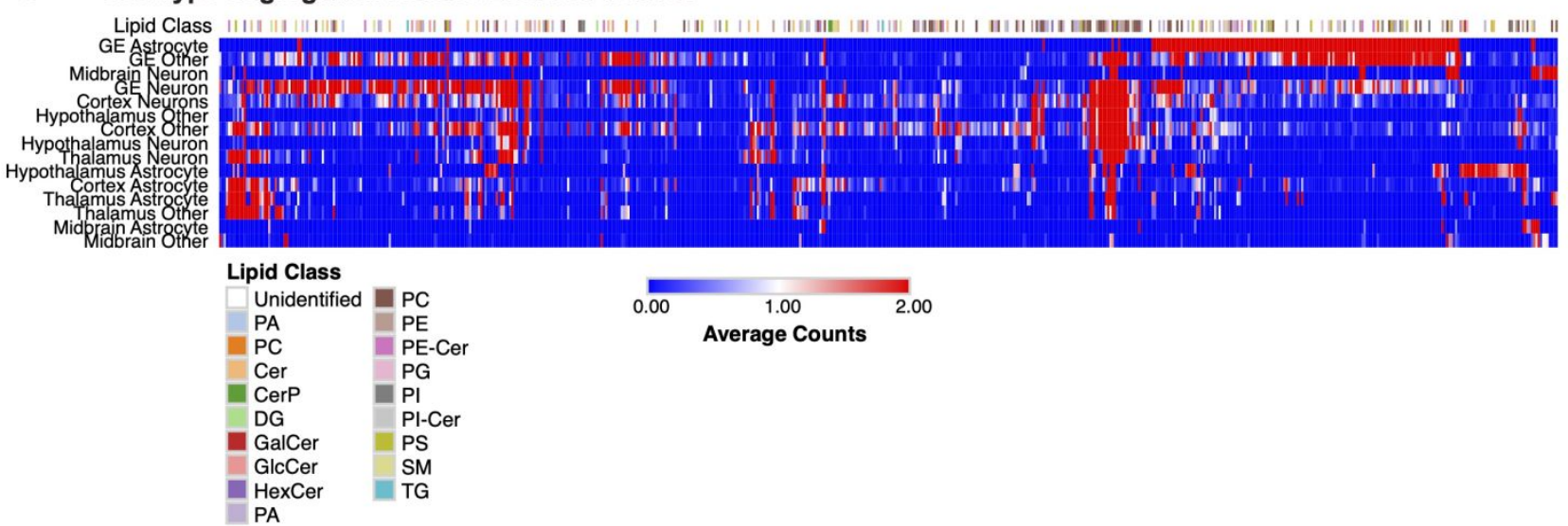

Figure

S5. Lipid Based Classification of Cell Class Across Brain Structures. A) Hierarchically clustered (across rows and columns) heatmap of each cortical area with lipid intensities for each of the 600 lipids averaged across cell class (neuron, astrocyte, or other). This clustering brings together the neuronal populations, and area-specific signatures are observable in the lipid signatures, consistent with previously reported transcriptomic data. B) Hierarchically clustered (across rows and columns) heatmap of each brain structure with lipid intensities for each of the 600 lipids averaged across cell class (neuron, astrocyte, or other). When identifiable, the lipid class is colored above. Structure-specific signatures are observable in the lipid signatures, consistent with our transcriptomic data. 
A Single-Cell Lipidomics Browser Overview
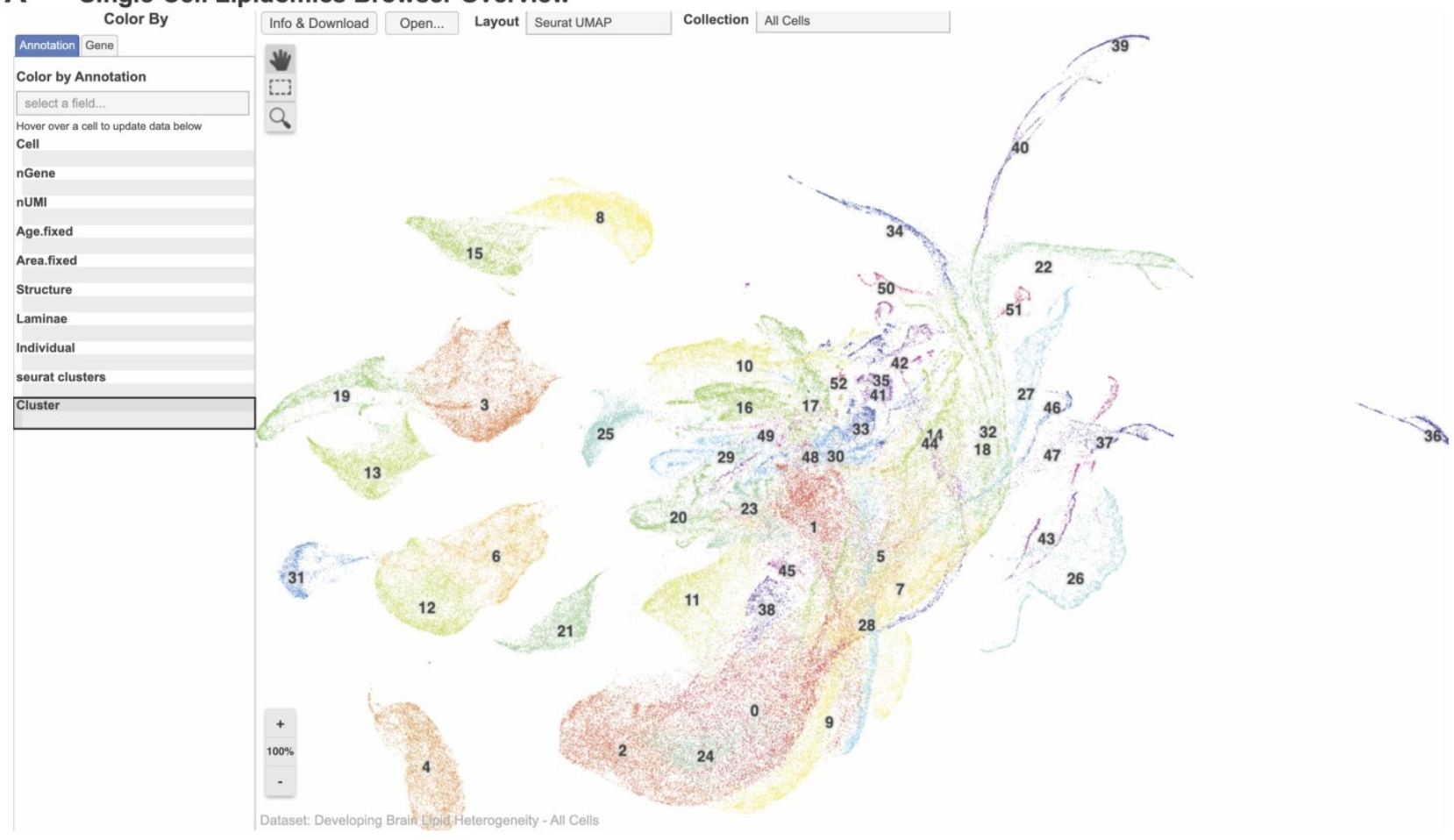

B Data Download and Cluster Markers

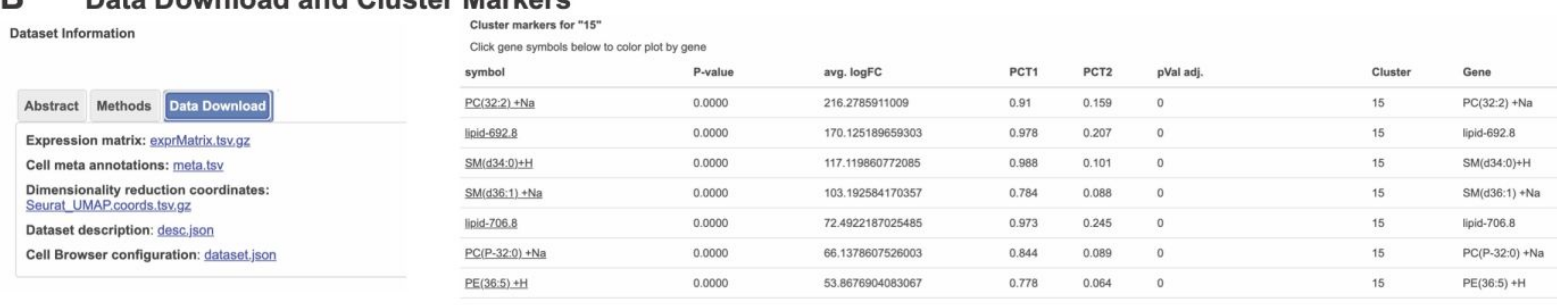

\section{Feature Plots}
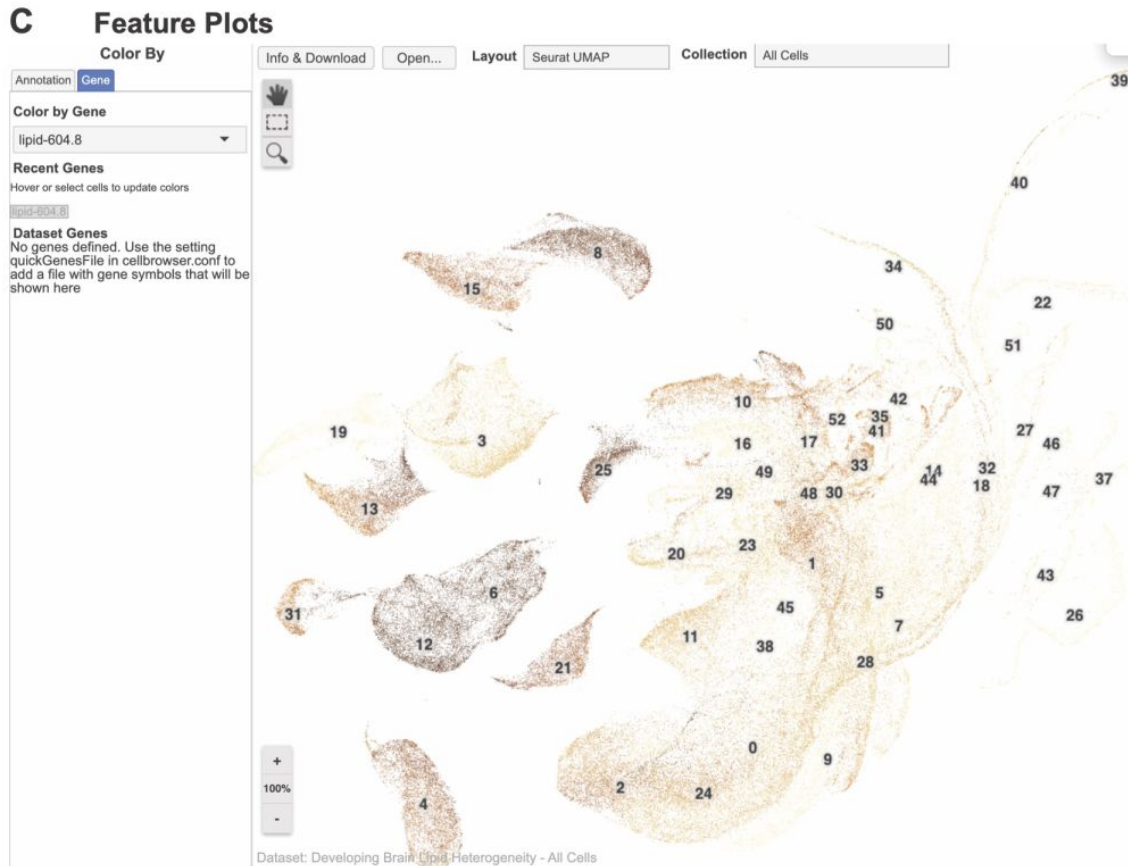

Figure S6. Single-cell Lipidomics Browser. A) A screenshot from the front page of the lipidomics browser. Clicking on any of the meta-data properties enables coloring of the plot by that underlying data. The screenshot here is the whole dataset, but from the front page one can open either this dataset or the cortex only dataset. B) Clicking on the Info and Download button enables you to go to this detailed page from which the matrices used to do the analysis can be retrieved, as can any relevant metadata. On the right, a 
screenshot is shown that appears when clicking on a cluster. The contents of this table include the lipid markers, annotated by lipid identity if known. C) Clicking on the "gene" tab from the main screen allows coloring of the UMAP plot by intensity of lipid content, which can be typed in or selected from the dropdown menu. After creating a feature plot by this lipid, the right panel will show the legend and the overall expression in a violin plot. 